\title{
Single-Layer Bandpass Active Frequency Selective Surface
}

Ghaffer I. Kiani , Kenneth L. Ford, Karu P. Esselle, Andrew R. Weily, C. Panagamuwa, John C. Batchelor

This is the peer reviewed version of the following article (Ghaffer I. Kiani , Kenneth L. Ford, Karu P. Esselle, Andrew R. Weily, C. Panagamuwa, John C. Batchelor, Single-Layer Bandpass Active Frequency Selective Surface, Wiley, Microwave and Optical Technology Letters, 50, (8), August 2008, pp. 2149-2151), which has been published in final form at (http://dx.doi.org/10.1002/mop.23615).

This article may be used for non-commercial purposes in accordance with Wiley Terms and Conditions for self-archiving 
Single-Layer Bandpass Active Frequency Selective Surface

Ghaffer I. Kiani , Kenneth L. Ford, Karu P. Esselle, Andrew R. Weily, C. Panagamuwa, John C. Batchelor

Abstract: A single-layer, bandpass, active FSS is presented. It shows good angle of incidence stability for TE incidence, at the operating frequency of $2.45 \mathrm{GHz}$. It is based on circular loop aperture with each unit cell having four PIN diodes. A novel method for dc biasing is used. About $12 \mathrm{~dB}$ average variation in transmission loss, between ON and OFF states, has been experimentally achieved at 2.45 $\mathrm{GHz}$.

Keywords: Active, frequency selective surface (FSS), oblique incidence, security, wireless networks.

\section{Introduction}

The use of frequency selective surfaces (FSS) as spatial filters from microwave to submillimeter wave frequencies is quite common [1]. Recently, a considerable amount of research is carried out in the field of active FSS to achieve a reconfigurable frequency response for different applications [2-6]. Among different methods to achieve a variable FSS frequency response, the most common is the use of PIN diodes to switch an FSS between ON and OFF states. Most of the previous research in this area has been carried out on bandstop FSS, such as arrays of metallic dipoles, which have switching devices (e.g. diodes) placed across the dipoles that can be varied via an external stimulus. Conversely, their bandpass versions consisting of arrays of slots are not suitable for the inclusion of active elements, as the bias applied to the devices will short across the metal surface. Recently, an attempt was made to design an active band pass FSS using two FSS layers [6]. In this design, one of the FSS layers is a standard bandstop active FSS which is placed in front of a passive bandpass FSS with a thin layer of PCB. This design is quite complex and hence needs accurate design tools and high manufacturing accuracy. In order to alleviate these constraints, a single layer bandpass active FSS is presented in this paper.

\section{Active FSS Modelling}

The front and the rear views of the new FSS unit cell are shown in the inset of Figs. 1. and 2. A circular aperture is used as the FSS element. Four diodes are placed orthogonal to each other on a unit cell of FSS. The outer and the inner edge diameters of the circular loop aperture are $26.6 \mathrm{~mm}$ and $25.6 \mathrm{~mm}$, respectively. This makes the width of aperture $0.5 \mathrm{~mm}$. The periodicity of the FSS elements is $30 \mathrm{~mm}$. The inter-element spacing is kept small to enhance angle of incidence stability [1]. Negative dc biasing is provided from the reverse side of the dielectric substrate by means of symmetrical cross-shaped bias lines. Their width is $0.5 \mathrm{~mm}$. The metallic circular plate on the front side of unit cell is connected to the centre of the cross shaped negative bias lines with the help of through pin having a diameter of $0.5 \mathrm{~mm}$. The pin is soldered on both sides to provide an electrical connection. The positive dc biasing is provided from the outer end of circular loop aperture on the front side of FSS. 


\section{PIN Diode}

The diode modelling is a crucial issue for which research into several diodes was carried out and the diode with the suitable specifications (BAP51-03) was chosen. The criteria for suitable specification were the size, the cost and the least forward resistance of the PIN diode. The equivalent circuit model of the PIN diode is derived from the work done in [6]. The leakage resistance in the OFF state is neglected in our design for simplicity. The typical values used for forward bias (ON) are $R=5 \Omega$ and $\mathrm{L}=0.4 \mathrm{nH}$, while for reverse bias (OFF) a series capacitance of $0.27 \mathrm{pF}$ is added to the circuit model [6]. During measurements, a forward voltage of $1 \mathrm{~V}$ was applied to the FSS prototype to switch PIN diodes $\mathrm{ON}$, while no voltage was applied in the reverse bias.

\section{Theoretical and Measured Results}

The FSS unit cell was modelled in CST Microwave Studio using periodic boundary conditions. Fig. 1 depicts the results of the simulation showing the transmission and reflection characteristics of the single-layer active FSS at $0^{\circ}, 30^{\circ}$ and $45^{\circ}$ incident angles for TE incidence when the PIN diodes are in OFF state. The resonance occurs at $2.45 \mathrm{GHz}, 2.46 \mathrm{GHz}$ and $2.47 \mathrm{GHz}$ for $0^{\circ}, 30^{\circ}$ and $45^{\circ}$ incidence, respectively. As far as the transmission is concerned, a small insertion loss of $0.5 \mathrm{~dB}$ can be noticed for all incident angles considered. Fig. 2 shows the transmission and reflection characteristics of the single layer active FSS in ON state. The circular apertures are effectively shorted together by the conducting diodes, resulting in an effective conducting sheet. There is no noticeable change in the reflection properties as the angle of incidence is increased from $0^{\circ}$ to $45^{\circ}$ degrees while the transmission coefficients changed slightly $(-17.8 \mathrm{~dB}$ to $-21.2 \mathrm{~dB}$ at $2.45 \mathrm{GHz})$. Theoretically, an average transmission loss of $19 \mathrm{~dB}$ can be achieved at the resonance frequency.

The single layer active FSS has been fabricated on a $420 \times 270$ mm FR4 sheet ( $15 \times 9$ elements) with dielectric constant of 4.4 and $1.6 \mathrm{~mm}$ thickness. Fig. 3 shows the measured transmission and reflection characteristics at $0^{\circ}, 30^{\circ}$ and $45^{\circ}$ degree incident angles for TE incidence when the PIN diodes are in OFF state. The resonance occurs at $2.63 \mathrm{GHz}, 2.64 \mathrm{GHz}$ and $2.65 \mathrm{GHz}$, respectively, for $0^{\circ}, 30^{\circ}$ and $45^{\circ}$ incidence, while the transmission loss at these angles are $2.7 \mathrm{~dB}, 2.8 \mathrm{~dB}$ and $3.4 \mathrm{~dB}$, respectively. The reason for higher resonance is the extra inductance contributed by the connecting $\mathrm{dc}$ lines at the end of FSS prototype. Moreover, the main causes for transmission loss are dielectric losses, diode losses, the reflection losses due to physical presence of diodes and the soldering material on FSS surface. Fig. 4 depicts the measured transmission and reflection coefficients for TE incidence when the diodes are in $\mathrm{ON}$ state. For $0^{\circ}, 30^{\circ}$ and $45^{\circ}$ incident angles, the reflection coefficient is almost $0 \mathrm{~dB}$ while the transmission loss is $13.1,14.8 \mathrm{~dB}$ and $15.8 \mathrm{~dB}$, respectively. Therefore, at $0^{\circ}, 30^{\circ}$ and $45^{\circ}$ angles of incidence, the transmission loss can be varied by $10.4 \mathrm{~dB}, 12$ $\mathrm{dB}$ and $12.4 \mathrm{~dB}$, respectively, by switching the PIN diodes from OFF to ON state.

This active FSS design is not stable for parallel polarisation (TM). Research is being carried out to address this issue which will be published elsewhere.

\section{Conclusion}

In this paper, a single-layer bandpass active FSS is presented. The design has shown a stable transmission response for oblique TE incidence in both ON and OFF states. An additional average transmission loss of about $12 \mathrm{~dB}$ has been experimentally achieved for the angles of incidence 
considered, by switching PIN diodes between forward and reverse bias. A further isolation can be obtained by using a dielectric with a low loss tangent and better quality PIN diodes. Beside other applications, it may find use in the intelligent Spectrum Selective Island (ISSI) applications.

\section{References}

[1] B. A. Munk, Frequency Selective Surfaces: Theory and Design. New York: Wiley, 2000.

[2] T.K. Chang, R.J. Langley and E.A. Parker, Active Frequency Selective Surfaces, IEE Proc., Part H, 143, Feb 1996, pp.62-66.

[3] T.K. Chang, R.J. Langley and E.A. Parker, An active square loop Frequency Selective Surface, IEEE Microwave and Guided Wave Letters, Vol. 3, No. 10, Oct. 1993.

[4] B.M. Cahill, E.A. Parker, "Field switching in an enclosure with active FSS screen", IEE Electronics Letters, Vol. 37, No. 4, Feb. 2001.

[5] B. Philip, E.A. Parker and R.J. Langley, Active FSS in an experimental horn antenna switchable between two beamwidths, IEE Electronics Letters, Vol. 31, No. 1, Jan. 1995.

[6] K. Mitchell, A. Keen, L. Davenport, C. Smartt, P. Leask and R. Larson, J. Davies, Research to demonstrate the ability of close-coupled frequency selective structures to enhance the spectral efficiency of radio systems in buildings, Ofcom Project Report AY4462B, (www.ofcom.org.uk/research/technology/spectrum_efficiency_scheme/ses200304/ay4462b/ay446 2b1_1.pdf)

\section{Figures captions}

Fig. 1. Theoretical transmission and reflection characteristics of the single-layer active FSS when the diodes are in OFF state. In the inset, the front view of the active FSS unit cell.

Fig. 2. Theoretical transmission and reflection characteristics of the single-layer active FSS when the diodes are in ON state. In the inset, the rear view of the active FSS unit cell.

Fig. 3. Measured transmission and reflection characteristics of the single-layer active FSS when the diodes are in OFF state.

Fig. 4. Measured transmission and reflection characteristics of the single-layer active FSS when the diodes are in ON state. 


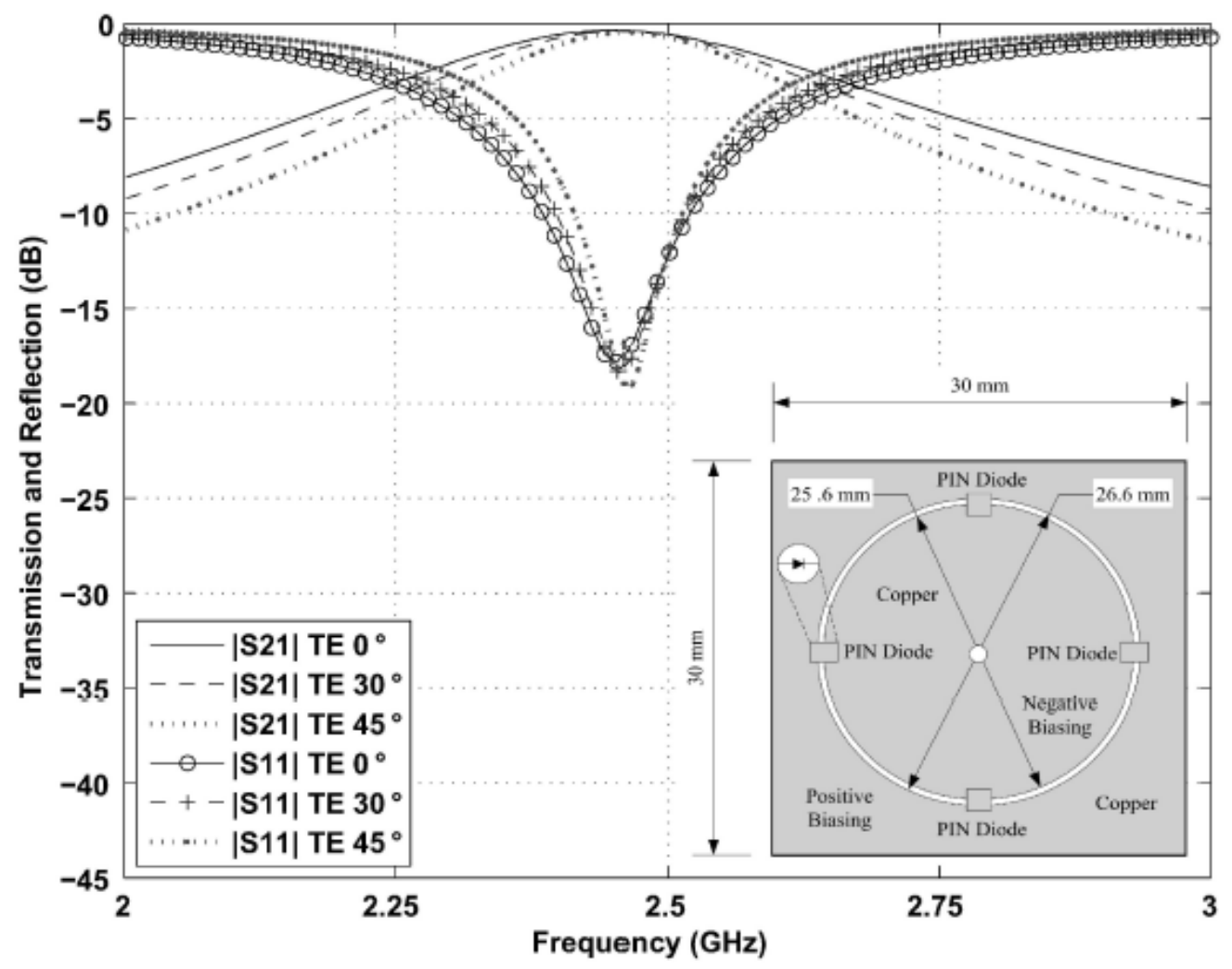

Theoretical transmission and reflection characteristics of the single-layer active FSS when the diodes are in OFF state. In the inset, the front view of the active FSS unit cell. $178 \times 141 \mathrm{~mm}(600 \times 600 \mathrm{DPI})$

Figure 1. 


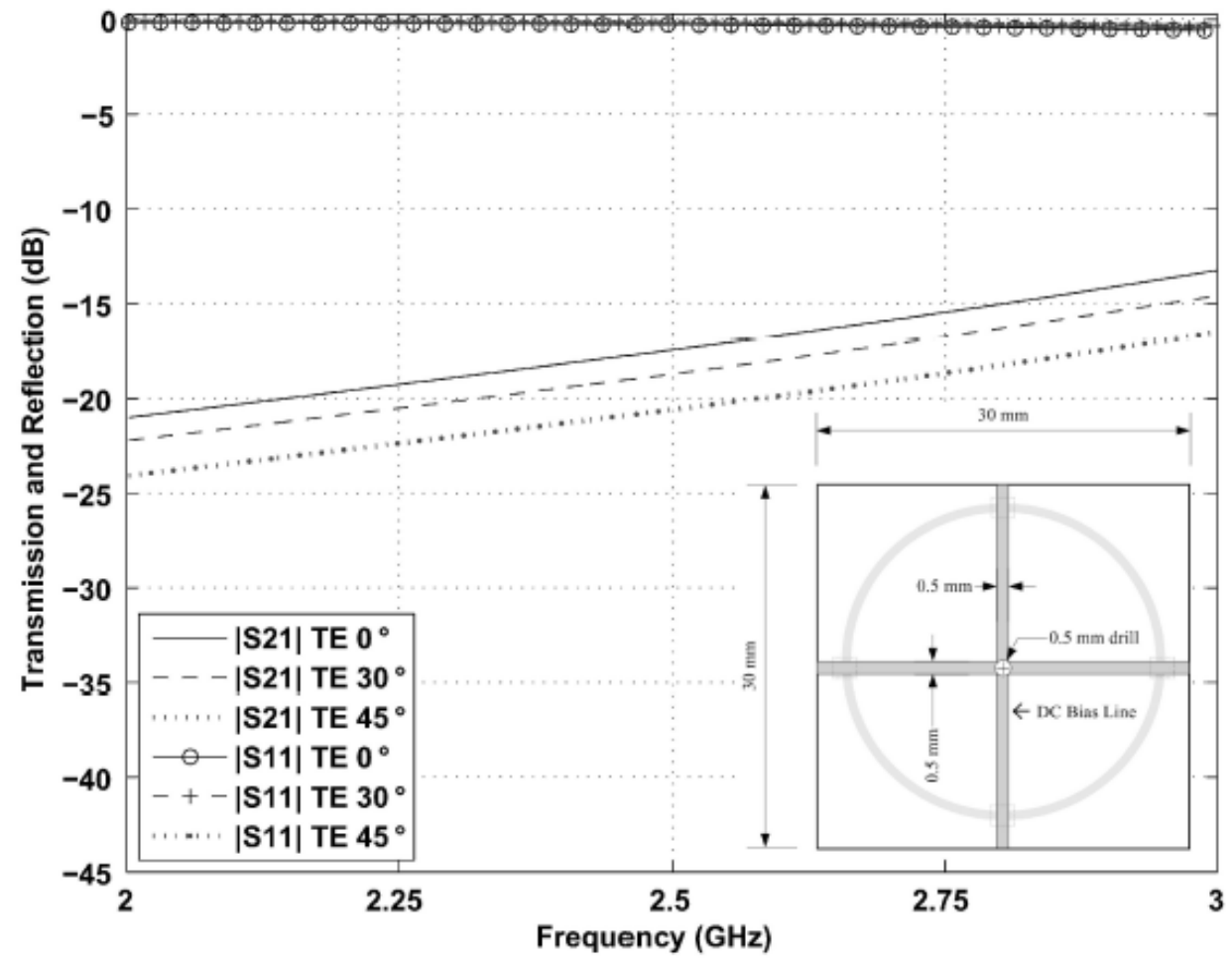

Theoretical transmission and reflection characteristics of the single-layer active FSS when the diodes are in ON state. In the inset, the rear view of the active FSS unit cell. $178 \times 141 \mathrm{~mm}(600 \times 600 \mathrm{DPI})$

Figure 2. 


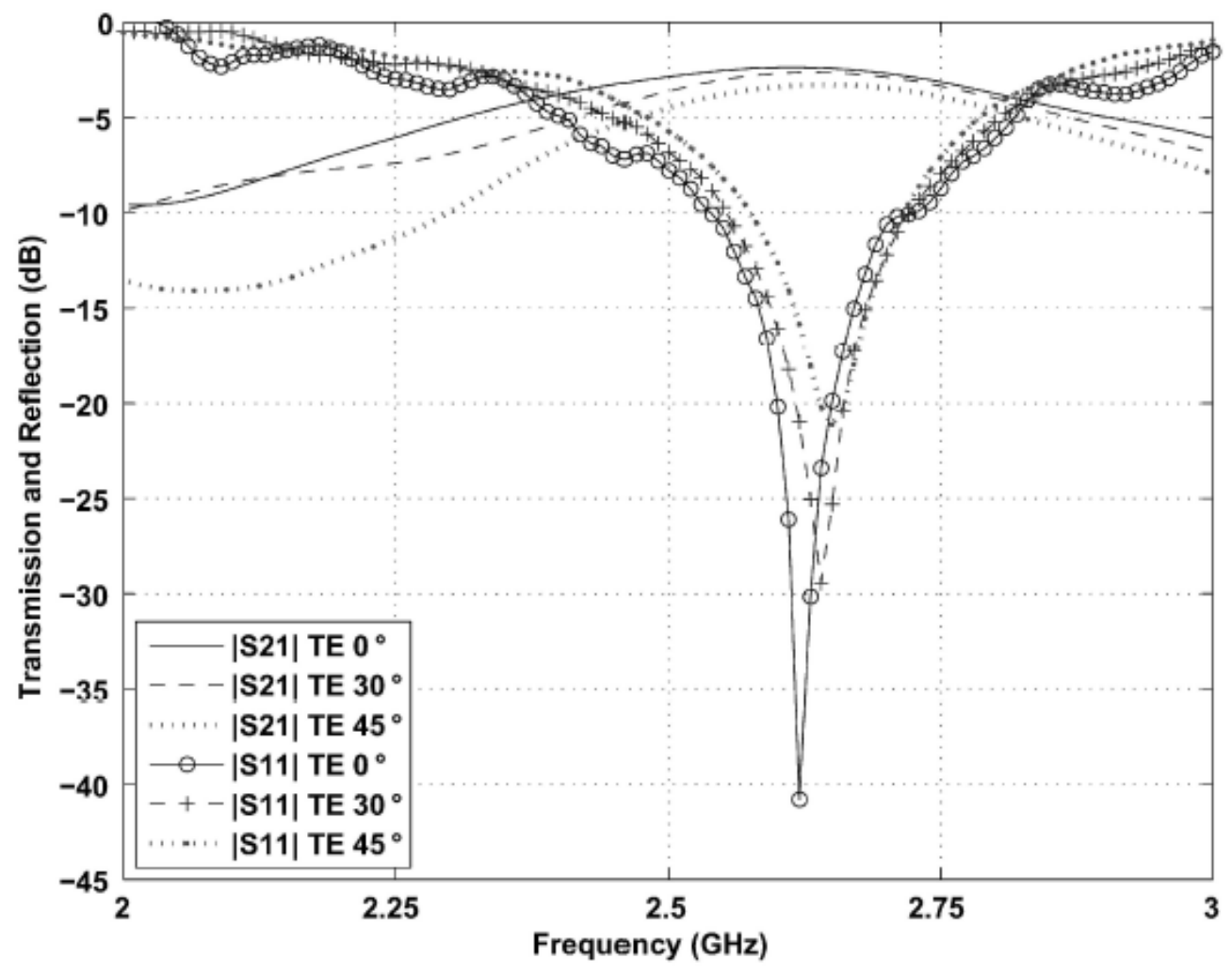

Measured transmission and reflection characteristics of the single-layer active FSS when the diodes are in OFF state.

$178 \times 141 \mathrm{~mm}(600 \times 600 \mathrm{DPI})$

Figure 3. 


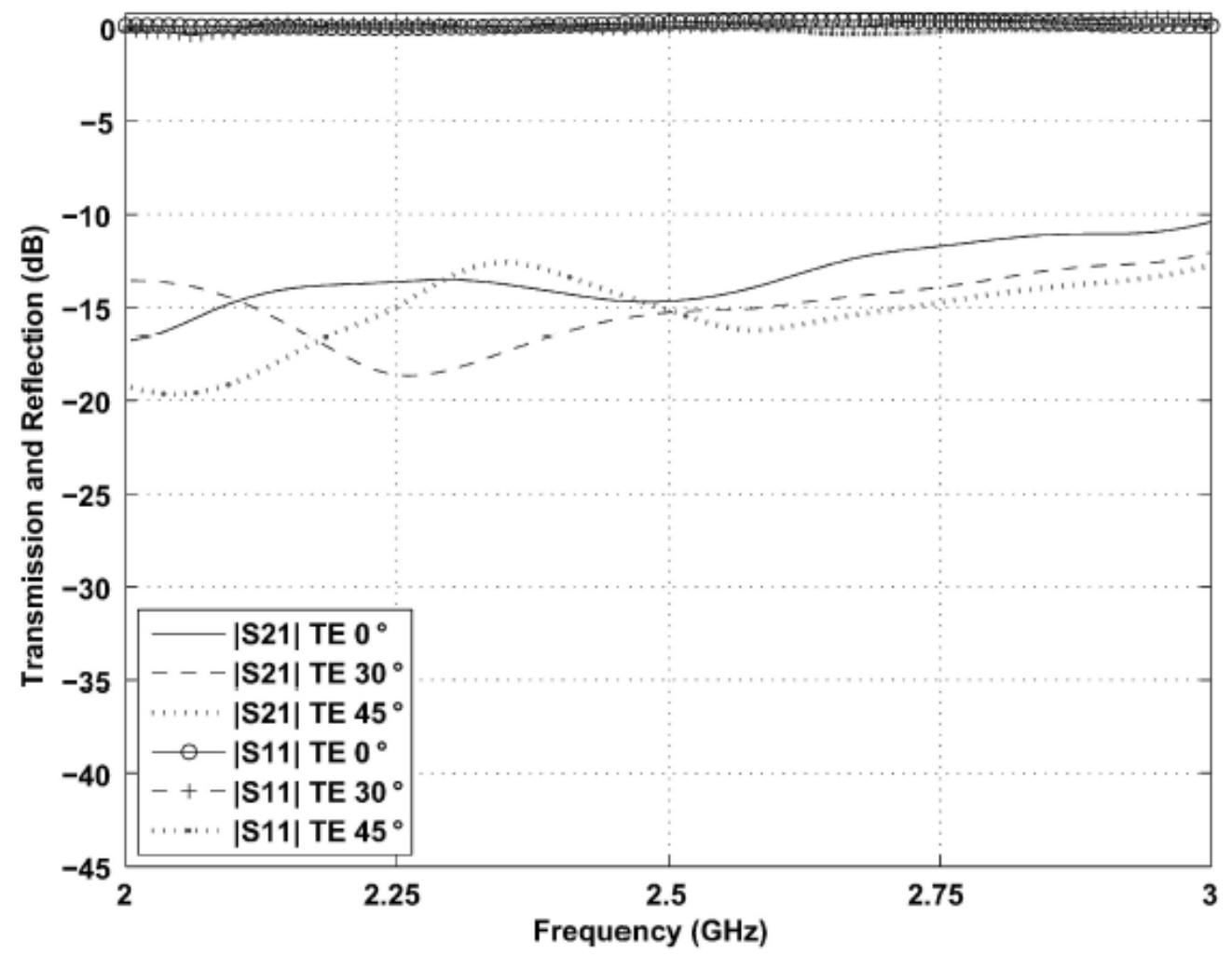

Measured transmission and reflection characteristics of the single-layer active FSS when the diodes are in $\mathrm{ON}$ state.

$178 \times 141 \mathrm{~mm}(600 \times 600 \mathrm{DPI})$

Figure 4 